\title{
Spin conductance, dynamic spin stiffness and spin diffusion in itinerant magnets
}

\author{
Peter Kopiet团 \\ Institut für Theoretische Physik der Universität Göttingen, \\ Bunsenstrasse 9, D-37073 Göttingen, Germany
}

(August 25, 1997)

\begin{abstract}
We discuss analogies between the charge- and spin response functions of itinerant magnets. We show that the spin-analog of the charge stiffness is not given by the usual spin stiffness $\rho_{s}$, but by the dynamic spin stiffness $D_{s}$, which is obtained from the dynamic spin conductance $G_{s}(\omega)$ in the limit of vanishing frequency $\omega$. The low-frequency behavior of $G_{s}(\omega)$ is used to define ideal spin conductors, normal spin conductors, and spin insulators. Assuming diffusive spin dynamics, we show that the spin diffusion coefficient is proportional to $\lim _{\omega \rightarrow 0} \operatorname{Re} G_{s}(\omega)$. We exploit this fact to develop a new extrapolation scheme for the spin diffusion coefficient in the paramagnetic phase of the Hubbard model.
\end{abstract}

Typeset using REVTEX 


\section{INTRODUCTION}

In a classic paper on the Theory of the Insulating State W. Kohn [1] pointed out that the behavior of the charge stiffness $D_{c}$ can be used to distinguish the conducting from the insulating state of interacting electrons. Physically $D_{c}$ can be identified with the weight of the Drude peak of the frequency-dependent conductivity $\sigma(\omega)$. A system with a finite value of $D_{c}$ deserves to be called an ideal conductor, because then $\sigma(\omega)$ diverges as $D_{c} /(i \omega)$ for $\omega \rightarrow 0$. In contrast, a normal conductor does not have a Drude peak $\left(D_{c}=0\right)$, and the conductivity $\sigma(\omega)$ approaches a non-zero value for vanishing frequency. Finally, the insulating state can be characterized by $D_{c}=0$ and $\sigma(\omega=0)=0$. Note that $D_{c}$ is defined in terms of the conductivity, a dynamic quantity. Nevertheless, $D_{c}$ can be obtained without explicitly calculating $\sigma(\omega)$ : as shown by Kohn [1], the second derivative of the free energy with respect to a fictitious vector potential (which is equivalent to a twist in the boundary conditions on the many-body wave-function) is proportional to the charge stiffness. More recently, the arguments of Kohn [1] have been sharpened by Shastry and Sutherland [2], who calculated $D_{c}$ exactly for Heisenberg-Ising and Hubbard rings using the ansatz of Bethe.

Another important observable, which is closely related to $D_{c}$, is the superfluid stiffness $\rho_{c}$ (the motivation for our slightly unconventional notation will become obvious below). Whereas $D_{c}$ characterizes the normal conducting properties of the system, a finite value of $\rho_{c}$ indicates long-range superconducting correlations. $D_{c}$ and $\rho_{c}$ can both be obtained as different limits of the wave-vector and frequency-dependent current response function $K_{\alpha \beta}(\mathbf{q}, \omega)$, which is given by the Kubo formula [3]. We shall briefly summarize the relevant definitions in Sec.[1].

While $D_{c}$ and $\rho_{c}$ in Hubbard and related models have recently been studied numerically by several groups [3 5], the analogous quantities $D_{s}$ and $\rho_{s}$ that characterize the spin dynamics have not received much attention. In fact, some authors seem not to be fully aware of the physically different meaning of the spin analog $D_{s}$ of the charge stiffness on the one hand, and the spin analog $\rho_{s}$ of the superfluid stiffness on the other hand. The quantity 
$\rho_{s}$ is commonly called the spin stiffness, and is related to the change in energy due to a small static twist in the directions of the spins at the boundaries of the system [6]. A finite value of $\rho_{s}$ in the thermodynamic limit is a manifestation of (quasi)-long-range magnetic order. Obviously, the spin stiffness $\rho_{s}$ and the superfluid stiffness $\rho_{c}$ are analogous quantities: both measure the degree of off-diagonal long-range order in the system. But what is the spin analog of the charge stiffness, and what is its physical meaning? In this paper we shall not only answer this question, but also discuss the more general concept of the spin conductance $G_{s}(\omega)$. We show that the limiting behavior of $G_{s}(\omega)$ for vanishing frequency $\omega$ can be used to define ideal spin conductors, normal spin conductors, and spin insulators, in complete analogy with the charge transport. Normal spin conductors are of particular interest, because systems with diffusive spin dynamics fall into this category. In Sec.[V we shall use the concept of the spin conductance to develop a new extrapolation scheme for calculating the spin diffusion coefficient of these systems. For localized spin models (such as the Heisenberg model) we have discussed the spin conductance and its usefulness for the calculation of the spin diffusion coefficient in Ref. [7].

For definiteness we shall consider the repulsive Hubbard model, although our considerations are easily generalized for other itinerant magnets. The Hamiltonian is given by $\hat{H}=\hat{T}+\hat{V}$, with

$$
\begin{aligned}
& \hat{T}=-t \sum_{\mathbf{r}} \sum_{\alpha=1}^{d}\left[c_{\mathbf{r}}^{\dagger} c_{\mathbf{r}+\mathbf{a}_{\alpha}}+\text { h.c. }\right] \\
& \hat{V}=U \sum_{\mathbf{r}}\left[c_{\mathbf{r} \uparrow}^{\dagger} c_{\mathbf{r} \uparrow}-\frac{1}{2}\right]\left[c_{\mathbf{r} \downarrow}^{\dagger} c_{\mathbf{r} \downarrow}-\frac{1}{2}\right],
\end{aligned}
$$

where $\mathbf{r}$ labels the $N$ sites of a $d$-dimensional hyper-cubic lattice, and $\mathbf{a}_{\alpha}$ are the primitive vectors with length $a$ in direction $\alpha=1, \ldots, d$. The operators $c_{\mathbf{r} \sigma}^{\dagger}, \sigma=\uparrow, \downarrow$ create spin- $\sigma$ electrons at lattice site $\mathbf{r}$, and $c_{\mathbf{r}}^{\dagger}=\left[c_{\mathbf{r} \uparrow}^{\dagger}, c_{\mathbf{r} \downarrow}^{\dagger}\right]$ are two-component operators. 


\section{CHARGE RESPONSE: CHARGE AND SUPERFLUID STIFFNESS}

Let us first recall the Kubo formula for the conductivity, which measures the linear charge response to an electromagnetic field. The usual frequency-dependent conductivity can be written as $\sigma_{\alpha \beta}(\omega)=\frac{e^{2}}{h} a^{2-d} G_{\alpha \beta}(\omega)$, where the dimensionless conductance $\left[\mathbb{b} G_{\alpha \beta}(\omega)\right.$ can be obtained from the current response function $K_{\alpha \beta}(\mathbf{q}, \omega)$,

$$
G_{\alpha \beta}(\omega)=\lim _{\mathbf{q} \rightarrow 0} \frac{K_{\alpha \beta}\left(\mathbf{q}, \omega+i 0^{+}\right)}{i\left(\hbar \omega+i 0^{+}\right)} .
$$

The current response function has two contributions,

$$
K_{\alpha \beta}\left(\mathbf{q}, \omega+i 0^{+}\right)=-\delta_{\alpha \beta} D^{\text {dia }}+P_{\alpha \beta}\left(\mathbf{q}, \omega+i 0^{+}\right),
$$

where the diamagnetic part $D^{\text {dia }}$ is proportional to the thermal expectation value of the kinetic energy operator,

$$
D^{\text {dia }}=\frac{1}{d N}\langle-\hat{T}\rangle \equiv \frac{1}{d N} \sum_{n} p_{n}\langle n|-\hat{T}| n\rangle .
$$

and the paramagnetic contribution is for general complex frequency $z$ given by

$$
\begin{aligned}
P_{\alpha \beta}(\mathbf{q}, z)=\frac{1}{N} \sum_{n, m} & p_{n}\left[\frac{\left\langle n\left|\hat{J}_{\alpha}(\mathbf{q})\right| m\right\rangle\left\langle m\left|\hat{J}_{\beta}(-\mathbf{q})\right| n\right\rangle}{E_{m}-E_{n}-\hbar z}\right. \\
& \left.+\frac{\left\langle n\left|\hat{J}_{\beta}(-\mathbf{q})\right| m\right\rangle\left\langle m\left|\hat{J}_{\alpha}(\mathbf{q})\right| n\right\rangle}{E_{m}-E_{n}+\hbar z}\right] .
\end{aligned}
$$

Here $|n\rangle$ denotes a complete set of exact eigenstates of $\hat{H}$, and $p_{n}=\left(\sum_{m} e^{-E_{m} / T}\right)^{-1} e^{-E_{n} / T}$ are the thermal occupation probabilities of states with energies $E_{n}$, where $T$ is the temperature (measured in units of energy). The current operators are

$$
\hat{J}_{\alpha}(\mathbf{q})=\frac{t}{2 i} \sum_{\mathbf{r}} e^{-i \mathbf{q} \cdot \mathbf{r}}\left[c_{\mathbf{r}}^{\dagger}\left(c_{\mathbf{r}+\mathbf{a}_{\alpha}}-c_{\mathbf{r}-\mathbf{a}_{\alpha}}\right)-\text { h.c. }\right] .
$$

The charge stiffness tensor $\left[D_{c}\right]_{\alpha \beta}$ and the superfluid stiffness tensor $\left[\rho_{c}\right]_{\alpha \beta}$ can be defined via the following limiting procedures,

$$
\begin{aligned}
{\left[D_{c}\right]_{\alpha \beta} } & =-\lim _{\omega \rightarrow 0}\left[\lim _{\mathbf{q} \rightarrow \mathbf{0}} K_{\alpha \beta}\left(\mathbf{q}, \omega+i 0^{+}\right)\right], \\
{\left[\rho_{c}\right]_{\alpha \beta} } & =-\lim _{\mathbf{q} \rightarrow 0}\left[\lim _{\omega \rightarrow 0} K_{\alpha \beta}\left(\mathbf{q}, \omega+i 0^{+}\right)\right] .
\end{aligned}
$$


For completeness, let us also introduce the q-dependent superfluid stiffness [9],

$$
\left[\rho_{c}(\mathbf{q})\right]_{\alpha \beta}=-\lim _{\omega \rightarrow 0} K_{\alpha \beta}\left(\mathbf{q}, \omega+i 0^{+}\right)
$$

which probes the response to time-independent transverse electromagnetic fields (see below). Eqs.(3), (8)-(10) relate physical quantities characterizing the charge dynamics to the appropriate limits of the linear response function $K_{\alpha \beta}(\mathbf{q}, \omega)$. For convenience, we have chosen a gauge where the scalar potential is set equal to zero, so that the electric field is represented by a vector potential, $\mathbf{E}(t)=-c^{-1} \partial \mathbf{A}(t) / \partial t$. This gauge, which is used very often in the literature, has the advantage that the current response can be expressed entirely in terms of the current-current correlation function. Of course, the physical current response is gauge invariant, see Ref. [10].

Quite generally, at long wavelengths $(|\mathbf{q}| a \ll 1)$ the current response tensor $K_{\alpha \beta}(\mathbf{q}, \omega)$ can be decomposed into a longitudinal part $\left(K_{\|}\right)$and a transverse part $\left(K_{\perp}\right)$, i.e.

$$
K_{\alpha \beta}(\mathbf{q}, \omega)=\hat{q}_{\alpha} \hat{q}_{\beta} K_{\|}(\mathbf{q}, \omega)+\left(\delta_{\alpha \beta}-\hat{q}_{\alpha} \hat{q}_{\beta}\right) K_{\perp}(\mathbf{q}, \omega)
$$

where $\hat{q}_{\alpha}=\hat{\mathbf{a}}_{\alpha} \cdot \hat{\mathbf{q}}$, with $\hat{\mathbf{a}}_{\alpha}=\mathbf{a}_{\alpha} / a$ and $\hat{\mathbf{q}}=\mathbf{q} / q$. The corresponding decomposition for the tensor $\left[\rho_{c}(\mathbf{q})\right]_{\alpha \beta}$ contains only a transverse component,

$$
\left[\rho_{c}(\mathbf{q})\right]_{\alpha \beta}=\left(\delta_{\alpha \beta}-\hat{q}_{\alpha} \hat{q}_{\beta}\right) \rho_{c}(\mathbf{q})
$$

The longitudinal part vanishes. Physically, this is due to the fact that a static longitudinal vector potential cannot induce any current [11. If we set $\mathbf{q}=0$ in Eq.(11) we have $K_{\perp}(0, \omega)=K_{\|}(0, \omega)$, because for a spatially uniform field the longitudinal and transverse response are identical. Thus, the conductance tensor $G_{\alpha \beta}(\omega)$ in Eq.(3) and the charge stiffness tensor $\left[D_{c}\right]_{\alpha \beta}$ in Eq.(8) are proportional to the unit matrix (for a system with cubic symmetry). The usual charge stiffness $D_{c}$ and superfluid stiffness $\rho_{c}$ can be identified with eigenvalues of the corresponding ternsors $(8,9)$, i.e. $\left[D_{c}\right]_{\alpha \beta}=\delta_{\alpha \beta} D_{c}$, and $\left[\rho_{c}\right]_{\alpha \beta}=\left(\delta_{\alpha \beta}-\hat{q}_{\alpha} \hat{q}_{\beta}\right) \rho_{c}$. Note that with our normalization both quantities have units of energy. $\rho_{c}$ is proportional to the density of the superconducting electrons. The finite value of $\rho_{c}$ in a superconductor is 
closely related to the screening of the magnetic field, i.e. the Meissner effect. Note that in a normal metal $\rho_{c}=0$, which is a consequence of the fact that in the normal metallic state static magnetic fields are not screened. Finally, let us point out that the physical meaning of the different order of limits in Eqs.(8) and (9) is easy to understand from the Maxwell equation $c \mathbf{q} \times \mathbf{E}(\mathbf{q}, \omega)=\omega \mathbf{B}(\mathbf{q}, \omega)$ : If we first take the limit $\omega \rightarrow 0$, the electric field $\mathbf{E}$ vanishes while the magnetic field $\mathbf{B}$ can remain finite - in this way we probe the Meissner effect. On the other hand, if we first let $\mathbf{q} \rightarrow 0$, we are left with an electric field.

\section{SPIN RESPONSE: DYNAMIC AND STATIC SPIN STIFFNESS}

The generalization of the above definitions for the spin degrees of freedom is straightforward. Denoting by $\sigma^{i}, i=x, y, z$, the Pauli matrices, in $d$ dimensions we may define $3 \times d$ spin-current operators,

$$
\begin{array}{r}
\hat{J}_{\alpha}^{i}(\mathbf{q})=\frac{t}{2 i} \sum_{\mathbf{r}} e^{-i \mathbf{q} \cdot \mathbf{r}}\left[c_{\mathbf{r}}^{\dagger} \frac{\sigma^{i}}{2}\left(c_{\mathbf{r}+\mathbf{a}_{\alpha}}-c_{\mathbf{r}-\mathbf{a}_{\alpha}}\right)-\text { h.c. }\right] \\
i=x, y, z \quad, \quad \alpha=1, \ldots, d
\end{array}
$$

In complete analogy with Eq.(4) we define the retarded spin-current response function

$$
K_{\alpha \beta}^{i j}\left(\mathbf{q}, \omega+i 0^{+}\right)=-\delta_{\alpha \beta} \delta_{i j} D^{\mathrm{dia}}+P_{\alpha \beta}^{i j}\left(\mathbf{q}, \omega+i 0^{+}\right)
$$

where the diamagnetic contribution $D^{\text {dia }}$ is given in Eq.(5), and the paramagnetic term $P_{\alpha \beta}^{i j}(\mathbf{q}, z)$ is simply obtained from Eq.(可) by replacing $\hat{J}_{\alpha} \rightarrow \hat{J}_{\alpha}^{i}$ and $\hat{J}_{\beta} \rightarrow \hat{J}_{\beta}^{j}$. As discussed by Chandra, Coleman, and Larkin [12], the spin-current response function $K_{\alpha \beta}^{i j}(\mathbf{q}, \omega)$ gives the spin response to a fictitious vector potential $\delta A_{\beta}^{i}(\mathbf{q}, \omega)$, which describes a space- and time-dependent modulation in the local spin-density. The proper definition of the dynamicand static spin stiffness tensor is now evident,

$$
\begin{aligned}
& {\left[D_{s}\right]_{\alpha \beta}^{i j} }=-\lim _{\omega \rightarrow 0}\left[\lim _{\mathbf{q} \rightarrow 0} K_{\alpha \beta}^{i j}\left(\mathbf{q}, \omega+i 0^{+}\right)\right], \\
& {\left[\rho_{s}\right]_{\alpha \beta}^{i j}=-\lim _{\mathbf{q} \rightarrow 0}\left[\lim _{\omega \rightarrow 0} K_{\alpha \beta}^{i j}\left(\mathbf{q}, \omega+i 0^{+}\right)\right] }
\end{aligned}
$$


Furthermore, in analogy with the q-dependent superfluid stiffness introduced in Eq.(10), let us define the q-dependent spin stiffness tensor [14]

$$
\left[\rho_{s}(\mathbf{q})\right]_{\alpha \beta}^{i j}=-\lim _{\omega \rightarrow 0} K_{\alpha \beta}^{i j}\left(\mathbf{q}, \omega+i 0^{+}\right)
$$

Finally, in analogy with Eq.(3) we introduce the dimensionless spin conductance

$$
\left[G_{s}(\omega)\right]_{\alpha \beta}^{i j}=\lim _{\mathbf{q} \rightarrow 0} \frac{K_{\alpha \beta}^{i j}\left(\mathbf{q}, \omega+i 0^{+}\right)}{i\left(\hbar \omega+i 0^{+}\right)}
$$

Because the operators $\hat{J}_{\alpha}^{i}(0)$ define the ferromagnetic spin-currents, the limit $\mathbf{q} \rightarrow 0$ in

Eqs.(15), (16) and (18) implies that we are looking at ferromagnetic correlations. In the case of antiferromagnetism we simply should consider the limit $\mathbf{q} \rightarrow \boldsymbol{\Pi}$ instead, where $\boldsymbol{\Pi}=$ $[\pi / a, \ldots, \pi / a]$ is the antiferromagnetic ordering wave-vector. A summary of the analogous quantities characterizing the charge- and the spin dynamics is given in Table @. We would like to emphasize that the spin analog of the Drude weight $D_{c}$ is given by the dynamic spin stiffness $D_{s}$, and not by the static spin stiffness $\rho_{s}$. It seems that the dynamic spin stiffness has not been discussed in the literature on the Hubbard model. Following the terminology used for the charge dynamics, a system with $D_{s}>0$ can be called an ideal spin conductor. For $D_{s}=0$ and $G_{s}(0) \neq 0$ the system is a normal spin conductor, and the spin insulator can be characterized by $D_{s}=G_{s}(0)=0$.

\section{SPIN DIFFUSION}

\section{A. Spin diffusion coefficient and Thouless number}

To see the connection between the spin conductance and spin diffusion, consider the dynamic structure factor for the spin degrees of freedom,

$$
\begin{aligned}
S^{i j}(\mathbf{q}, \omega) & =\frac{2 \pi \hbar}{N} \sum_{n, m} p_{n} \delta\left(E_{m}-E_{n}-\hbar \omega\right) \\
& \times\left\langle n\left|\hat{S}_{\mathbf{q}}^{i}\right| m\right\rangle\left\langle m\left|\hat{S}_{-\mathbf{q}}^{j}\right| n\right\rangle .
\end{aligned}
$$


Here the Fourier components of the spin operators are $\hat{S}_{\mathbf{q}}^{i}=\sum_{\mathbf{r}} e^{-i \mathbf{q} \cdot \mathbf{r}} c_{\mathbf{r}}^{\dagger} \frac{\sigma^{i}}{2} c_{\mathbf{r}}$. General hydrodynamic arguments [13] show that the diffusive spin dynamics manifests itself in the following long-wavelength and low-energy form of the dynamic structure factor,

$$
S^{i j}(\mathbf{q}, \omega)=2 \delta^{i j} \chi \frac{\hbar \omega}{1-e^{-\hbar \omega / T}} \frac{\mathcal{D} \mathbf{q}^{2}}{\omega^{2}+\left(\mathcal{D} \mathbf{q}^{2}\right)^{2}}
$$

where we have assumed cubic symmetry and spin-rotational invariance. Here $\mathcal{D}$ is the spin diffusion coefficient, and the static susceptibility $\chi$ is

$$
\chi=\frac{1}{T} \lim _{\mathbf{q} \rightarrow 0} \int_{-\infty}^{\infty} \frac{d \omega}{2 \pi} S^{i i}(\mathbf{q}, \omega)=\frac{1}{T} \sum_{\mathbf{r}}\left\langle\hat{S}_{0}^{i} \hat{S}_{\mathbf{r}}^{i}\right\rangle .
$$

On the other hand, the real part of the dimensionless spin conductance (18) is given by

$$
\operatorname{Re}\left[G_{s}(\omega)\right]_{\alpha \beta}^{i j}=-\pi \delta(\hbar \omega)\left[D_{s}\right]_{\alpha \beta}^{i j}+\left[G_{s}^{\prime}(\omega)\right]_{\alpha \beta}^{i j}
$$

where the weight of the $\delta$-function can be identified with the dynamic spin stiffness (15), and the paramagnetic contribution is

$$
\begin{aligned}
{\left[G_{s}^{\prime}(\omega)\right]_{\alpha \beta}^{i j}=} & \lim _{\mathbf{q} \rightarrow \mathbf{0}} \frac{\operatorname{Im} K_{\alpha \beta}^{i j}\left(\mathbf{q}, \omega+i 0^{+}\right)}{\hbar \omega} \\
= & \pi \frac{1-e^{-\hbar \omega / T}}{\hbar \omega} \lim _{\mathbf{q} \rightarrow 0} \frac{1}{N} \sum_{n, m} p_{n} \delta\left(E_{m}-E_{n}-\hbar \omega\right) \\
& \quad \times\left\langle n\left|\hat{J}_{\alpha}^{i}(\mathbf{q})\right| m\right\rangle\left\langle m\left|\hat{J}_{\beta}^{j}(-\mathbf{q})\right| n\right\rangle .
\end{aligned}
$$

The matrix elements of the current operator $\hat{\mathbf{J}}_{\alpha}=\left[\hat{J}_{\alpha}^{x}, \hat{J}_{\alpha}^{y}, \hat{J}_{\alpha}^{z}\right]$ can be related to the matrix elements of the spin operators $\hat{\mathbf{S}}_{\mathbf{q}}$ via the Heisenberg equation of motion. Using the fact that the Hubbard interaction is spin-rotationally invariant, it is easy so show that to leading order in $\mathbf{q} \cdot \mathbf{a}_{\alpha}$

$$
i \hbar \frac{\partial \hat{\mathbf{S}}_{\mathbf{q}}}{\partial t}=\left[\hat{\mathbf{S}}_{\mathbf{q}}, \hat{T}\right]=\sum_{\alpha=1}^{d}\left(\mathbf{q} \cdot \mathbf{a}_{\alpha}\right) \hat{\mathbf{J}}_{\alpha}(\mathbf{q})
$$

Hence,

$$
\left(E_{m}-E_{n}\right)\left\langle n\left|\hat{\mathbf{S}}_{\mathbf{q}}\right| m\right\rangle=\sum_{\alpha=1}^{d}\left(\mathbf{q} \cdot \mathbf{a}_{\alpha}\right)\left\langle n\left|\hat{\mathbf{J}}_{\alpha}(\mathbf{q})\right| m\right\rangle .
$$

Substituting this expression into Eq.(19), it is easy to show that 


$$
\left[G_{s}^{\prime}(\omega)\right]_{\alpha \alpha}^{i i}=\frac{1-e^{-\hbar \omega / T}}{\hbar \omega} \frac{\hbar}{2} \lim _{\mathbf{q} \rightarrow 0} \frac{\omega^{2}}{(\mathbf{q} a)^{2}} S^{i i}(\mathbf{q}, \omega) .
$$

Assuming now the diffusive form (20) of the dynamic structure factor, we obtain for the spin diffusion coefficient

$$
\frac{\hbar \mathcal{D}}{a^{2}}=\frac{1}{\chi} \lim _{\omega \rightarrow 0}\left[G_{s}^{\prime}(\omega)\right]_{\alpha \alpha}^{i i} .
$$

Moreover, it is not difficult to show [7] that in the presence of spin diffusion the dynamic spin stiffness $D_{s}$ vanishes due to a perfect cancellation between the dia- and paramagnetic contributions in Eq.(15). Thus, the existence of spin diffusion means that the system is a normal spin conductor.

Eq.(27) can be rewritten in a form which emphasizes a deep connection between spin diffusion and charge diffusion in disordered electronic systems [15, 16, 7]. Defining the rescaled dimensionless spin conductance [8]

$$
g_{s}=\left(\frac{L}{a}\right)^{d-2} \lim _{\omega \rightarrow 0}\left[G_{s}^{\prime}(\omega)\right]_{\alpha \alpha}^{i i},
$$

and the energies

$$
E_{\mathrm{Th}}=\frac{\hbar \mathcal{D}}{L^{2}} \quad, \quad \Delta_{s}=\frac{1}{N \chi},
$$

(where $L=a N^{1 / d}$ is the linear size of the system) we obtain from Eq.(27)

$$
g_{s}=\frac{E_{\mathrm{Th}}}{\Delta_{s}} .
$$

This expression should be compared with the well-known Thouless formula $g=E_{\mathrm{Th}} / \Delta$ for the dimensionless average conductance of a disordered electronic system. Here the so-called Thouless energy $E_{\mathrm{Th}}$ is defined as in Eq.(29) (with $\mathcal{D}$ now given by the average charge diffusion coefficient of the disordered system), and $\Delta$ is the average level spacing at the Fermi energy. Thus, Eqs.(27) and (30) are nothing but the Thouless formula for the spin diffusion problem. The dimensionless number $g_{s}$ defined in Eq.(30) is the corresponding Thouless number. In analogy with disordered electrons, a system with $g_{s} \gg 1$ can be called a spin metal. 


\section{B. Spin diffusion in the Hubbard model}

The above analogies are not only interesting from a formal point of view, but also useful in practice. We now show that Eq.(27) offers a new and physically transparent extrapolation scheme for directly calculating the spin diffusion coefficient of the Hubbard model. See Ref. [7] for a similar calculation for the Heisenberg model, and Ref. [17] for an alternative method to calculate the spin diffusion coefficient in the two-dimensional $t-J$-model.

After some straightforward manipulations, Eq.(27) can be cast into the form

$$
\frac{\hbar \mathcal{D}}{a^{2}}=\frac{t^{2}}{T \chi} \int_{0}^{\infty} d s C(s)
$$

where the correlation function $C(s)$ is given by

$$
C(s)=\frac{1}{2 N}\langle[\hat{I}(s)+\hat{I}(-s)] \hat{I}\rangle .
$$

Here $\hat{I}(s)=e^{i \hat{H} s} \hat{I} e^{-i \hat{H} s}$, and the (dimensionless) current operator $\hat{I}$ is

$$
\begin{aligned}
\hat{I} & =\frac{1}{2 i} \sum_{\mathbf{r}}\left[c_{\mathbf{r}}^{\dagger} \frac{\sigma^{z}}{2}\left(c_{\mathbf{r}+\mathbf{a}_{\alpha}}-c_{\mathbf{r}-\mathbf{a}_{\alpha}}\right)-\text { h.c. }\right] \\
& =\sum_{\mathbf{k}} \sin \left(k_{x} a\right)\left[c_{\mathbf{k} \uparrow}^{\dagger} c_{\mathbf{k} \uparrow}-c_{\mathbf{k} \downarrow}^{\dagger} c_{\mathbf{k} \downarrow}\right]
\end{aligned}
$$

where $c_{\mathbf{k}}=N^{-1 / 2} \sum_{\mathbf{r}} e^{-i \mathbf{k} \cdot \mathbf{r}} c_{\mathbf{r}}$. The bracket in Eq. (32) denotes thermal average with respect to the interacting Hamiltonian $\hat{H}=\hat{T}+\hat{V}$, see Eqs.(11) and (2). Because the kinetic energy

operator $\hat{T}$ commutes with the current operator $\hat{I}$, for $U=0$ the integral in Eq.(31) does not exist. Then our model is an ideal spin conductor. This is not surprising, because the diffusive dynamics in a system without disorder must be a correlation effect. We would like to emphasize that Eq.(31) has been derived under the assumption that the spin dynamics is diffusive. The divergence of the integral for $U=0$ simply indicates that in this case our assumption is not correct.

Because for $U=0$ the integral in Eq.(31) is infinite, we expect that for small $U$ the spin diffusion coefficient diverges with some power of $t / U$. Of course, for finite $U$ the correlator $C(s)$ cannot be calculated exactly, so that we have to make some physically 
motivated approximation. A standard approximation scheme, which has proven to be quite reliable for the calculation of the spin diffusion coefficient of the Heisenberg model at high temperatures [7, 18], is the Gaussian extrapolation of the short-time expansion of $C(s)$ to long times. Expanding $C(s)$ in powers of $s$,

$$
C(s)=\sum_{n=0}^{\infty} \frac{(-1)^{n} s^{2 n}}{(2 n) !} C_{2 n}
$$

the coefficients $C_{2 n}$ can be written in terms of multiple commutators. Because $C(-s)=$ $C(s)$, only even powers of $s$ appear. The first two coefficients are

$$
\begin{aligned}
C_{0} & =\left\langle\hat{I}^{2}\right\rangle, \\
C_{2} & =\langle\hat{I}[[\hat{I}, \hat{H}], \hat{H}]\rangle \\
& =\langle\hat{I}[[\hat{I}, \hat{V}], \hat{V}]\rangle,
\end{aligned}
$$

where we have used $[\hat{I}, \hat{T}]=0$ and $\langle\hat{I}\rangle=0$. Assuming that the higher coefficients are consistent with a Gaussian, the long-time extrapolation is

$$
C(s) \approx C_{0} \exp \left[-\frac{C_{2} s^{2}}{2 C_{0}}\right] .
$$

Then we obtain from Eq.(31)

$$
\frac{\hbar \mathcal{D}}{a^{2}}=\frac{t^{2}}{T \chi} \frac{C_{0}}{2}\left[\frac{2 \pi C_{0}}{C_{2}}\right]^{1 / 2}
$$

Note that so far we have not assumed that the interaction is small, so that Eqs.(35)-(38) are valid for arbitrary $U$. For simplicity, let us now evaluate the coefficients $C_{0}$ and $C_{2}$ in the limit $U \rightarrow 0$. Then the averages in Eqs.(35) and (36) are easily calculated with the help of the Wick-theorem. Specializing to the case of half filling, we obtain after a lengthy but straightforward calculation

$$
\begin{aligned}
& C_{0}=2 A_{d}(T), \\
& C_{2}=4 U^{2} A_{d}(T) B_{d}(T),
\end{aligned}
$$

where 


$$
\begin{aligned}
& A_{d}(T)=\frac{1}{N} \sum_{\mathbf{k}} \sin ^{2}\left(k_{x} a\right) f\left(\epsilon_{\mathbf{k}} / T\right)\left[1-f\left(\epsilon_{\mathbf{k}} / T\right)\right], \\
& B_{d}(T)=\frac{1}{N} \sum_{\mathbf{k}} f\left(\epsilon_{\mathbf{k}} / T\right)\left[1-f\left(\epsilon_{\mathbf{k}} / T\right)\right] .
\end{aligned}
$$

Here $f(x)=\left[e^{x}+1\right]^{-1}$ is the Fermi function, and the non-interacting energy dispersion in $d$ dimensions is $\epsilon_{\mathbf{k}}=2 d t \gamma_{\mathbf{k}}$, with $\gamma_{\mathbf{k}}=d^{-1} \sum_{\alpha=1}^{d} \cos \left(\mathbf{k} \cdot \mathbf{a}_{\alpha}\right)$. Away from half filling we should replace $\epsilon_{\mathbf{k}} \rightarrow \epsilon_{\mathbf{k}}-\mu$, where $\mu$ is the chemical potential. In the non-interacting limit $T \chi=\frac{1}{2} B_{d}(T)$, so that we finally obtain from Eq.(38) in the limit $U \ll t$

$$
\frac{\hbar \mathcal{D}}{a^{2}}=2 \sqrt{\pi} \frac{A_{d}(T)}{B_{d}^{3 / 2}(T)} \frac{t^{2}}{U}
$$

Note that $\mathcal{D}$ diverges for $U \rightarrow 0$, in agreement with the fact that without correlations there is no spin diffusion. Obviously, the spin diffusion coefficient cannot be calculated by naive perturbation theory in powers of $U$.

In the limit $T \gg t$ we may use $A_{d}(\infty)=\frac{1}{4}$ and $B_{d}(\infty)=\frac{1}{8}$, so that Eq.43) reduces to

$$
\frac{\hbar \mathcal{D}}{a^{2}}=2 \sqrt{\pi} \frac{t^{2}}{U}, T=\infty
$$

independent of the dimensionality of the system. Recall that this result has been derived in the weak coupling limit. More generally, at $T=\infty$ it is easy to see from Eqs.(31), (32), and (34) that $\hbar \mathcal{D} / a^{2}$ is proportional to $t^{2} / U$ for all values of $U$. This follows from the fact that the expansion (34) of $C(s)$ is actually an expansion in powers of $(U s)^{2}$, because the current operator $\hat{I}$ commutes with the kinetic energy operator $\hat{T}$. Assuming that the integral is convergent, we may scale out the $U$-dependence by re-defining the integration variable $s^{\prime}=U s$. This leads trivially to the energy scale $t^{2} / U$. We would like to point out that at $T=\infty$ the spin diffusion coefficients of the spin $S=1 / 2$ quantum Heisenberg antiferromagnet and the half filled Hubbard model at strong coupling are not identical, although both are proportional to $t^{2} / U$ (see Refs. [19,7]). The reason is that for $T=\infty$ the value of $\mathcal{D}$ in the Hubbard model is determined by states with energies larger than $U$, while the mapping to the Heisenberg model is only valid for energy scales smaller than $U$. Only in the interval $t^{2} / U \ll T \ll U$ the half filled Hubbard model at strong coupling 
can be expected to have the same spin diffusion coefficient as the corresponding Heisenberg antiferromagnet with exchange coupling $J=4 t^{2} / U$.

Let us now discuss the low-temperature limit $T \ll t$. Using the fact that for $T \rightarrow 0$

$$
f\left(\epsilon_{\mathbf{k}} / T\right)\left[1-f\left(\epsilon_{\mathbf{k}} / T\right)\right] \rightarrow \frac{T}{2 d t} \delta\left(\gamma_{\mathbf{k}}\right),
$$

it is easy to see that Eq.(43) reduces to

$$
\frac{\hbar \mathcal{D}}{a^{2}}=2 \sqrt{\pi} \frac{a_{d}}{b_{d}^{3 / 2}}\left[\frac{2 d t}{T}\right]^{1 / 2} \frac{t^{2}}{U} \quad, \quad T \ll t,
$$

where the numerical constants $a_{d}$ and $b_{d}$ are

$$
\begin{aligned}
& a_{d}=\frac{1}{N} \sum_{\mathbf{k}} \sin ^{2}\left(k_{x} a\right) \delta\left(\gamma_{\mathbf{k}}\right), \\
& b_{d}=\frac{1}{N} \sum_{\mathbf{k}} \delta\left(\gamma_{\mathbf{k}}\right), \quad d \neq 2 .
\end{aligned}
$$

In $d=2$ the integral in Eq.(48) is logarithmically divergent (for $N \rightarrow \infty$ ), so that at low temperatures $b_{2}$ is given by

$$
b_{2} \approx \frac{4}{\pi^{2}} \ln (4 t / T) .
$$

Because we have assumed that the system is in the paramagnetic state, Eq.(46) should be valid for temperatures above the magnetic ordering temperature $T_{N}$. Keeping in mind

that in $d \leq 2$ there is no long range order at any finite temperature, and that in $d>2$ the ordering temperature $T_{N}$ is exponentially small at weak coupling, Eq.46) describes the low-temperature behavior of the spin diffusion coefficient in a wide range of temperatures that are small compared with the band-width $4 d t$. Although the precise numerical value of the prefactor in Eqs.(43) and (46) depends on our Gaussian extrapolation scheme, the energy scale $t^{2} / U$ in Eq.(43) and the low-temperature behavior given in Eq.(46) should be independent of the details of the extrapolation method.

\section{SUMMARY AND CONCLUSIONS}

In this work we have used analogies between charge and spin response functions of itinerant magnets to clarify the concept of the static and dynamic spin stiffness. Starting 
from the general Kubo formula for the relevant linear response functions, we have shown that the dynamic spin stiffness $D_{s}$ is the precise spin analog of the charge stiffness $D_{c}$. The usual (static) spin stiffness $\rho_{s}$ is the spin analog of the superfluid stiffness $\rho_{c}$, and is only finite in the presence of long-range magnetic order. Considering the fact that the charge stiffness has recently received a lot of attention [3] 5], it is rather surprising that the corresponding quantity $D_{s}$ has not been studied. Partially, this might be due to the fact that $D_{s}$ has often been confused with the static spin stiffness $\rho_{s}$. Table [ I summarizes analogous quantities. We would like to emphasize again that a finite value of $D_{s}$ does not imply the existence of long-range magnetic order. A simple example is the Hubbard model for $U=0$, where $D_{s}>0$ but $\rho_{s}=0$. A value $D_{s}>0$ simply means that the system is an ideal spin conductor, so that the spin transport is not diffusive. The analogy with charge transport is obvious: an ideal conductor has a finite charge stiffness $D_{c}>0$, implying an infinite conductivity. But a perfect conductor is not necessarily a superconductor. Only in the latter case $\rho_{c}>0$.

The low-frequency behavior of the dynamic spin conductance $G_{s}(\omega)$ can be used to classify the spin dynamics into three categories: ideal spin conductors, normal spin conductors, and spin insulators. In Sec. $\mathbb{\nabla}$ we have further analyzed a particular class of normal spin conductors, namely systems with diffusive spin dynamics. In this case the dynamic spin stiffness vanishes, but the spin conductance $G_{s}(\omega)$ has a finite limit for vanishing frequency, which is proportional to the spin diffusion coefficient. The concept of the spin conductance and the associated Thouless number offers a new and physically transparent extrapolation scheme for calculating the spin diffusion coefficient.

We hope that our work will stimulate further research in this field. Numerical calculations of $G_{s}(\omega)$ and $D_{s}$ in strongly correlated itinerant magnets are called for. In particular, by varying some external parameter (such as temperature, doping, or interaction strength), it might be possible to observe transitions between the three types of spin transport discussed above. Numerical calculations of the temperature-dependence of the spin conductance might also give evidence for spin-charge separation in strongly correlated systems: Very recently Si [20] pointed out that the existence of spin-charge separation manifests itself in different 
temperature-dependencies of the spin- and charge conductances.

\section{ACKNOWLEDGMENTS}

I would like to thank H. G. Evertz for a discussion during a workshop on the Role of Dimensionality in Correlated Electronic Systems at Villa Gualino (Torino, Italy), which eventually motivated me to write this paper. This work was partially supported by a Heisen-

berg Fellowship of the Deutsche Forschungsgemeinschaft, and by the ISI Foundation and EU HC\&M Network ERBCHRX-CT920020. 


\section{REFERENCES}

* Address from October 1997 - April 1998: Department of Physics and Astronomy, University of California, Los Angeles, California 90095.

[1] W. Kohn, Phys. Rev. 133, A171 (1964).

[2] B. Shastry and B. Sutherland, Phys. Rev. Lett. 65, 243 (1990).

[3] D. J. Scalapino, S. R. White and S. C. Zhang, Phys. Rev. Lett. 68, 2830 (1992).

[4] H. Castella, X. Zotos, and P. Prelovšek, Phys. Rev. Lett. 74, 972 (1995).

[5] X. Zotos and P. Prelovšek, Phys. Rev. B 53, 983 (1996).

[6] M. E. Fisher, M. N. Barber, and D. Jasnow, Phys. Rev. A 8, 1111 (1973); J. Rudnick and D. Jasnow, Phys. Rev. B 16, 2032 (1978).

[7] P. Kopietz, Mod. Phys. Lett. B 7, 1747 (1993).

[8] For a system of linear size $L$ the conductivity $\sigma$ is related to the usual dimensionless conductance $g$ via $\sigma=\left(e^{2} / h\right) L^{2-d} g$. Thus, our function $G_{\alpha \beta}$ in Eq.(3) is related to the dimensionless conductance $g$ via $G=(L / a)^{2-d} g$.

[9] See, for example, M. Tinkham, Introduction to Superconductivity, (Mc Graw-Hill, New York, 1975), chapter 2.10 .

[10] P. C. Martin, Measurements and Correlation Functions, in Many Body Physics, Les Houches 1967, edited by C. DeWitt and R. Balian, (Gordon and Breach, New York, 1968).

[11] D. Pines and P. Nozières, The Theory of Quantum Liquids, Vol. 1 , (Addison-Wesley Advanced Book Classics, Redwood City, 1989), chapter 4.7.

[12] P. Chandra, P. Coleman, and A. I. Larkin, J. Phys. Cond. Mat. 2, 7933 (1990).

[13] D. Forster, Hydrodynamic Fluctuations, Broken Symmetry, and Correlation Functions, 
(Benjamin, Reading, 1975).

[14] For an explicit calculation of $\rho_{s}(\mathbf{q})$ for two-dimensional quantum Heisenberg ferromagnets see P. Kopietz and G. E. Castilla, Phys. Rev. B 43, 11100 (1991). For Heisenberg antiferromagnets see S. Chakravarty, B. I. Halperin, and D. R. Nelson, Phys. Rev. B 39, 2344 (1989). We are not aware of any microscopic calculation of $\rho_{s}(\mathbf{q})$ for itinerant magnets.

[15] D. J. Thouless, Phys. Rep. 13, 93 (1974).

[16] S. Chakravarty, Phys. Rev. Lett. 66, 481 (1991).

[17] J. Bonča and J. Jaklič, Phys. Rev. B 51, 16083 (1995).

[18] T. Moriya, Prog. Theor. Phys. 16, 23 (1956); R. R. P. Singh and M. P. Gelfand, Phys. Rev. B 42, 996 (1990).

[19] P. G. De Gennes, J. Phys. Chem. Solids 4, 223 (1958); N. Mori and K. Kawasaki, Prog. Theor. Phys. 27, 529 (1962); H. S. Bennett and P. C. Martin, Phys. Rev. A 138, 608 (1965); A. G. Redfield and W. N. Yu, Phys. Rev. 169, 443 (1967); T. Morita, Phys. Rev. B 6, 3385 (1972).

[20] Q. Si, Phys. Rev. Lett. 78, 1767 (1997). 


\section{TABLES}

TABLE I. Analogous quantities characterizing charge- and spin dynamics.

\begin{tabular}{|c|c|c|}
\hline Charge & Spin & Physical meaning \\
\hline charge response function $K_{\alpha \beta}(\mathbf{q}, \omega)$ & spin response function $K_{\alpha \beta}^{i j}(\mathbf{q}, \omega)$ & $\begin{array}{l}\text { linear current response to an external } \\
\text { vector potential }\end{array}$ \\
\hline conductance $G_{\alpha \beta}(\omega)$ & spin conductance $\left[G_{s}(\omega)\right]_{\alpha \beta}^{i j}$ & $\begin{array}{l}\text { response to a time-dependent, spa- } \\
\text { tially constant vector potential }\end{array}$ \\
\hline q-dependent superfluid stiffness ${ }^{\mathrm{a}}\left[\rho_{c}(\mathbf{q})\right]_{\alpha \beta}$ & $\mathbf{q}$-dependent spin stiffness $\left[\rho_{s}(\mathbf{q})\right]_{\alpha \beta}^{i j}$ & $\begin{array}{l}\text { response to a time-independent, spa- } \\
\text { tially varying vector potential }\end{array}$ \\
\hline superfluid stiffness $\rho_{c}$ & spin stiffness $\rho_{s}$ & $\begin{array}{l}\text { probes long-range correlations (super- } \\
\text { conducting or magnetic) }\end{array}$ \\
\hline charge stiffness ${ }^{\mathrm{b}} D_{c}$ & dynamic spin stiffness $D_{s}$ & $\begin{array}{l}\text { finite for ideal (charge or spin) } \\
\text { conductor }\end{array}$ \\
\hline
\end{tabular}

an the book by Tinkham [9] the transverse eigenvalue of this tensor is denoted by $K(\mathbf{q})$.

${ }^{\mathrm{b}}$ Also known as Drude weight 